\title{
Generation of topologically complex three-dimensional electron beams in a plasma photocathode
}

\author{
Xinlu Xu๑, ${ }^{1,}$ ' Jorge Vieira, ${ }^{2}$ Mark J. Hogan, ${ }^{1}$ Chan Joshi, ${ }^{3}$ and Warren B. Mori $\odot^{3,4}$ \\ ${ }^{1}$ SLAC National Accelerator Laboratory, Menlo Park, California 94025, USA \\ ${ }^{2}$ GOLP/Instituto de Plasma e Fusão Nuclear, Instituto Superior Técnico, \\ Universidade de Lisboa, 1049-001 Lisbon, Portugal \\ ${ }^{3}$ Department of Electrical Engineering, University of California, Los Angeles, California 90095, USA \\ ${ }^{4}$ Department of Physics and Astronomy, University of California Los Angeles, \\ Los Angeles, California 90095, USA
}

(Received 29 September 2021; accepted 13 December 2021; published 21 January 2022)

\begin{abstract}
Laser-triggered ionization injection is a promising way of generating controllable high-quality electrons in plasma-based acceleration. We show that ionization injection of electrons into a fully nonlinear plasma wave wake using a laser pulse comprising of one or more Laguerre-Gaussian modes with combinations of spin and orbital angular momentum can generate exotic three-dimensional spatial distributions of highquality relativistic electrons. The phase dependent residual momenta and initial positions of the ionized electrons are encoded into their final phase space distributions, leading to complex spatiotemporal structures. The structures are formed as a result of the transverse (betatron) and longitudinal (phase slippage and energy gain) dynamics of the electrons in the wake immediately after the electrons are injected. Theoretical analysis and three-dimensional simulations verify this mapping process leads to the generation of these complex topological beams. These beams may trigger novel beam-plasma interactions as well as produce coherent radiation with orbital angular momentum when sent through a resonant undulator.
\end{abstract}

DOI: 10.1103/PhysRevAccelBeams.25.011302

Plasma-based acceleration (PBA) $[1,2]$ is attractive because it can provide acceleration gradients in excess of $\mathrm{GV} / \mathrm{cm}$. The last several decades have seen tremendous progress in PBA [3], including the demonstration of high gradients [4-6] and the generation of electron beams suitable for applications, e.g., driving a compact freeelectron-laser [7] and advanced QED studies [8,9], producing bright and collimated X-rays [10] for high energy density science [11] and imaging applications [12]. The production of high-quality electron beams [13-17] from controllable injection schemes [18-27] has been instrumental for these advances. Recent work on plasma cathodes has opened the possibility of generating femtosecond duration electron beams with peak currents as high as hundreds of kA $[28,29]$ and normalized emittance $\epsilon_{n}$ as low as 10's of nm [13,14,16,17,30-32].

Besides having the potential to achieve unprecedented beam brightness, plasma cathodes can imprint multidimensional spatial structures onto the accelerated beams.

\footnotetext{
*xuxinlu@slac.stanford.edu

Published by the American Physical Society under the terms of the Creative Commons Attribution 4.0 International license. Further distribution of this work must maintain attribution to the author(s) and the published article's title, journal citation, and DOI.
}

For instance, several schemes purport to generate longitudinally bunched (1D) electrons [33-37] with potential to produce temporally coherent radiation [38]. Electron rings have been observed in experiments from electron trapping within wake pockets created by sheath splitting [39]. Simulations have predicted that such electron rings can also be generated in donut shaped wakefields driven by relativistic higher order Laguerre-Gaussian (LG) laser pulses with normalized vector potentioal $a_{L}>1$ [40], where $a_{L} \equiv 8.6 \times 10^{-10} \lambda[\mu \mathrm{m}] I^{1 / 2}\left[\mathrm{~W} / \mathrm{cm}^{2}\right]>1$ and $\lambda, I$ are the wavelength and intensity of the laser pulse.

Producing electron beams with complicated topology through self-injection in nonlinear plasma waves is both of fundamental [41-44] and practical interest. Helically or sinusoidally modulated beams could be used to achieve superradiant emission of broadband X-rays in conventional and plasma-based light sources [45]. Furthermore, relativistic beams with nontrivial topologies could potentially emit coherent radiation with orbital angular momentum (OAM) [46,47], beyond the visible spectrum [48]. Shortwavelength OAM (vortex) light is interesting because it can extend the OAM laser-matter interactions to the nanometer or even atomic scale thereby enable interesting applications in many fields [49-54]. However, current laser plasmabased schemes produce electron beams with spiral structures by transferring a large amount of angular momentum to them through the twisted wakefield [44] or the twisted 
electromagnetic fields of a super-intense laser $\left(a_{L} \sim 100\right)$ with high-order LG mode [55-57]. These beams are characterized by large emittance and almost continuous energy spectrum, and are therefore not suitable for producing coherent radiation.

In this paper, we show through theory and supporting particle-in-cell (PIC) simulations that a plasma cathode based on ionization injection into a nonlinear wakefield from nonrelativistic lasers $\left(a_{L} \sim 0.1\right)$ with combinations of spin and orbital angular momentum can generate highquality electron beams with exotic three-dimensioanl (3D) spiral spatial distributions. The intensity of the ionizing laser needs to be only marginally above ionization thresholds, $\sim 10^{17} \mathrm{~W} / \mathrm{cm}^{2}$, thus such configuration is realizable with standard technology. It is now well appreciated that a circularly polarized $(C P)$ laser carries spin angular momentum while a linearly polarized LG mode carries OAM [46]. An OAM mode can be described as $\vec{A}=-\operatorname{Re}\left(\sigma \vec{e}_{x}+\right.$ $\left.i \hat{e}_{y}\right) a_{L} c_{p}^{|l|}(r, \theta, z) \mathrm{e}^{i(l \theta-k z+\omega t)}$ where the angular momentum per photon is $l \hbar$ [46], and $\sigma=1$ for right-handed $C P$ and -1 for left-handed. The details for the complex functions $c_{p}^{|l|}$ are given in the Appendix A.

In ionization injection electrons are born insider a fully blown out plasma wake through tunnel ionization of the electric field from one or more laser pulses. The 3D phase information of the laser at the instant of ionization is imprinted onto the final electron distribution when the electrons become trapped after the laser pulse eventually overtakes them. The 3D spatial distributions evolve spatiotemporally within the wake as the electrons gain energy and phase slip longitudinally while executing betatron oscillations under the transverse focusing force of the ion column in the nonlinear wake. Although ionization injection has been extensively studied, there has been no investigation into how the spin and OAM of the laser is imprinted onto the angular momentum and distribution of the self-injected electrons. Here, we show that this concept permits designing beams with complex spatiotemporal distributions. The electrons generated in this new scheme are characterized by small emittance $(\sim 100 \mathrm{~nm})$, small energy spread $(\sim 1.5 \mathrm{MeV})$, zero net angular momentum with a small spread $(\sim 100 \mathrm{~nm} \cdot m c)$, and $\mathrm{kA}$ current that are suitable to produce high power short wavelength radiation with OAM, where $m$ is the electron mass and $c$ is the speed of light in vacuum. By using multiple laser pulses with different polarizations and LG modes, a beam with an axially varying spiral structure or multiple beams with different twisted structures can be produced.

To illustrate how this injection scheme can be used to generate spiraling and other complex 3D structured beams, we consider a fully blown out wake generated by an electron beam driver. We simulate the ionization injection by an appropriately delayed but comoving ultrashort laser using nonevolving forces characteristic of nonlinear wakefields using the 3D PIC code OSIRIS [58,59]. The forces for electrons with forward velocity $\beta_{z} c$ are $F_{z}=\frac{\xi}{2} m \omega_{p}^{2}, F_{r}=-\left[\frac{r}{2}+\left(1-\beta_{z}\right) \frac{r}{2}\right] m \omega_{p}^{2}$, where $\xi \equiv c t-$ $z$ and $r$ are the longitudinal and transverse coordinates and $\omega_{p}$ is the ambient plasma frequency. This significantly reduces the computational requirements as we only need to follow the injected particles. This approximation is well justified as the wake created by a highly relativistic beam driver evolves very slowly (hundreds of plasma periods for $\mathrm{GeV}$-class drivers) on the time scales of the injection process (several plasma periods).

A CP $800 \mathrm{~nm}$ laser pulse with specified LG modes propagates through a mixture of majority hydrogen plasma with $n_{p}=1.74 \times 10^{17} \mathrm{~cm}^{-3}$ and minority $\mathrm{He}^{1+}$ plasma with a density of $10^{-4} n_{p}$. The injected electrons are supplied via laser ionization of the $\mathrm{He}^{1+}$ ions [60]. The density of $\mathrm{He}^{1+}$ is set to be low to minimize the space charge repulsion between the ionized electrons when they have low energies. The lasers are focused at $z=2 \frac{c}{\omega_{p}}$ with a spot size $w_{0}=0.22 \frac{c}{\omega_{p}}(2.8 \mu \mathrm{m})$ and start at $z=-2 \frac{c}{\omega_{p}}$ with a duration $\tau_{\mathrm{FWHM}}=0.23 \omega_{p}^{-1}(9.8 \mathrm{fs})$. The intensities of the pulses are adjusted to ensure similar injected charge in all cases: $a_{L}=0.085$ for $(l=0, p=0)$ while $a_{L}=0.14$ for other cases. The $\mathrm{He}^{1+}$ plasma starts from $z=-2 \frac{c}{\omega_{p}}$ to ensure that ionization within a Rayleigh length is included.

Simulation results are presented in Fig. 1 (see Appendix B and $\mathrm{C}$ for details on the simulation parameters and more cases). We only consider $l \geq 0$ without loss of generality. Density isosurfaces of the trapped electrons are shown in the first column while their density distributions in the $(\theta, \xi)$ plane are shown in the second column where $\theta \equiv \operatorname{atan} 2(y, x)[61]$ is the angle in the transverse plane. As is clear, electrons with complex 3D structures are formed. When using a right-handed $C P$ laser $(\sigma=1)$ with a fundamental LG mode $(l=0, p=0)$, a single spiral beam (corkscrew) is produced; when $(l=1, p=0)$, two spiraling beamlets twist around each other; when $(l=2, p=0)$, three beamlets twist together to form a triple helical structure; when a left-handed $C P$ laser $(\sigma=-1)$ with $(l=$ $1, p=0)$ is used, the spiral structure is absent altogether with the resulting beam forming a series of hollow shells. In the first two cases, the angle $\theta$ has an approximately linear dependence on the longitudinal position $\xi$ while this dependence is absent in the last case. The beams have $\epsilon_{n} \sim$ $100 \mathrm{~nm}$ and $\sim 1.5 \mathrm{MeV}$ uncorrelated energy spread (see Appendix D for their longitudinal phase space). Their energies at this time are $\sim 20 \mathrm{MeV}$ and can be boosted to $\mathrm{GeV}$-class in the following acceleration.

As shown in Fig. 1, column 3, we introduce a bunching factor $b\left(k, l_{b}\right)=\frac{1}{N}\left|\sum_{j=1}^{N} \exp \left[i\left(l_{b} \theta_{j}-k \xi_{j}\right)\right]\right|$ to quantify the 3D structures, where $N$ is the number of the electrons. For the beam produced by the $l=0$ laser, the bunching factor is maximum at $\left(l_{b}=1, k \approx 1.3 k_{L}\right)$ and the beam is rich in spatial harmonics, i.e., $\left(l_{b}=2, k \approx 2.6 k_{L}\right)$, 

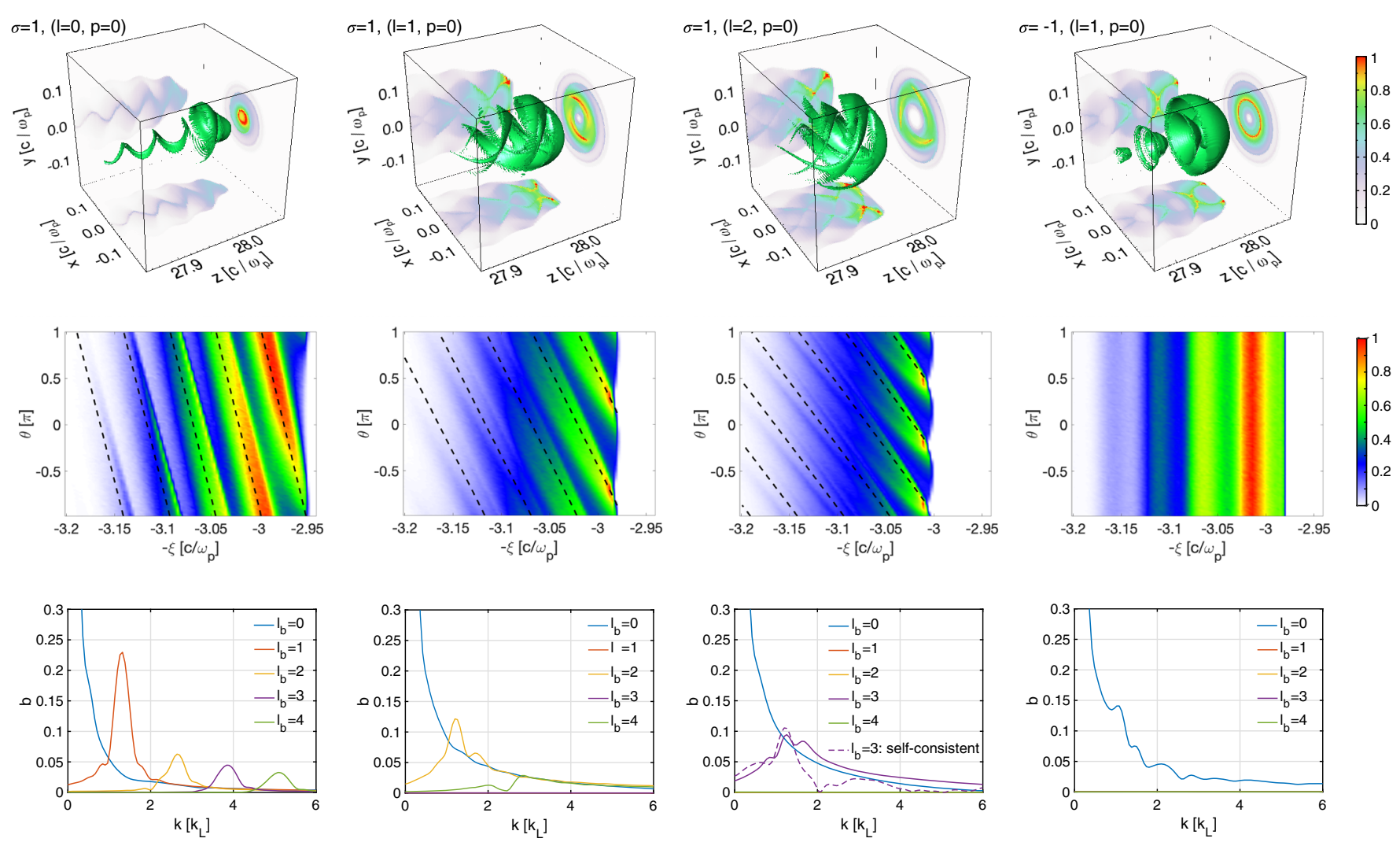

FIG. 1. The structures of the injected electrons at $\omega_{p} t=31$. First row: Isosurface of the electron density and its projections on each plane. Second row: Normalized density distribution of the electrons in the $\theta-\xi$ plane. The black dashed lines represent the predications from Eq. (2). Third row: bunching factor.

$\left(l_{b}=3, k \approx 3.9 k_{L}\right)$, and $\left(l_{b}=4, k \approx 5.2 k_{L}\right)$. When a laser with $l=1$ is used, the bunching factor achieves its maximum at $\left(l_{b}=2, k \approx 1.5 k_{L}\right)$ and the second harmonic appears at $l_{b}=4, k \approx 3 k_{L}$. When a laser with $l=2$ is used, the bunching factor is maximum at $\left(l_{b}=3, k \approx 1.5 k_{L}\right)$ while the harmonics are not distinctly present at this propagation time. A self-consistent simulation where an electron beam driver excites the wake and ionization of $n_{\mathrm{He}^{1+}}=0.05 n_{p}$ provides the injected electrons is shown by the dashed line. The high current $(2.5 \mathrm{kA})$ injected beam with $11.3 \mathrm{pC}$ charge has a similar bunching factor for $l_{b}=3$ which validates the nonevolving force model and shows the structure is still formed even for kA currents. In reality, a gas mixture of hydrogen and helium can be used while the electron of hydrogen and the first electron of helium can be ionized by the beam driver and/or a separate low intensity ionization laser. Thus the density of $\mathrm{He}^{1+}$ which is formed by ionization of the first helium electron can be adjusted by controlling the density of helium atoms while the background electron density is controlled by the ratio of hydrogen to helium.

When the polarization direction of the $l=1$ laser is reversed from right-handed to left-handed, the bunching factor is zero for all $l_{b} \neq 0$. Interestingly, the beams at this time are hollow which may be used to generate plasma wakes suitable for positron acceleration $[40,62]$ and clean the halo from heavy ion beams in conventional particle accelerators [63].

To understand the simulation results, we propose a model for the dynamics of the ionized electrons. After being tunnel-ionized, the electrons begin to move under the influence of the laser field and the plasma wakefield. When studying the longitudinal dynamics, the axial oscillations inside the laser pulse can be ignored since the energy gain from the low-intensity laser is negligible compared to that due to the wake. There is thus a longitudinal mapping between initial longitudinal position $\xi_{i}$ and the final position $\xi$ as described in Ref. [30]. In the case of a highly relativistic wake such as that produced by a $\mathrm{GeV}$ class e-bunch, the electrons ionized inside the wake first slip back but are accelerated by the longitudinal electric field of the wake, until they gain sufficient energy to move nearly synchronously (phase locked) with the wake longitudinally. This nearly locked position can be calculated using the form of the acceleration gradient inside the wake [30,34] as $k_{p} \xi \approx \sqrt{4+\left(k_{p} \xi_{i}\right)^{2}}$. Longitudinal mixing happens, i.e., electrons released at different time can reside in the same final slice [30]. 
The transverse motion of the injected electrons can be divided into two stages. In the first stage, after ionization, the electrons respond to the oscillating laser field. Since the field amplitude of the laser $\left(\sim 10 \frac{m c \omega_{p}}{e}\right)$ is typically much higher than the local value of the wakefield $\left(\sim 0.1 \frac{m c \omega_{p}}{e}\right)$, the electrons can be assumed to oscillate only under the influence of the laser field. The electrons are rapidly passed over by the ionizing laser since their longitudinal velocities are much less than $c$, and they then conduct betatron oscillations in the wakefield [64].

The transverse canonical momentum $\vec{P}_{\perp} \equiv \vec{p}_{\perp}-e \vec{A}_{\perp}$ is conserved if a plane wave assumption is made. In the first stage, so long as $k_{L} w_{0} \gg 1$ then canonical momentum remains approximately satisfied for LG modes. We have verified the conservation of $\vec{P}_{\perp}$ for the parameters used in the simulations by carrying out test particle simulations using OSIRIS (see Appendix E). Thus, the momenta of the electrons when they leave the laser pulse are equal to the vector potential at the instant of ionization. For a $C P$ laser pulse of a single LG mode, the normalized vector potential are approximately $a_{x} \approx-\sigma a(r) \cos (l \theta-\hat{\xi})$, $a_{y} \approx a(r) \sin (l \theta-\hat{\xi})$, where $\hat{\xi}=k_{L} \xi$. The transverse coordinates are assumed to be not changed during the transit time of the lasers [65].

In the second stage, the electrons begin to respond to the wakefield. Assuming each electron experiences a constant acceleration gradient $E_{z}$ and its energy increases adiabatically, the asymptotic solution of the equation of motion is [30]

$$
\begin{aligned}
& x \approx\left(\frac{1}{\gamma}\right)^{\frac{1}{4}} x_{i} \cos \Phi+\left(\frac{4}{\gamma}\right)^{\frac{1}{4}} \frac{p_{x i}}{m c k_{p}} \sin \Phi \text { and } \\
& y \approx\left(\frac{1}{\gamma}\right)^{\frac{1}{4}} y_{i} \cos \Phi+\left(\frac{4}{\gamma}\right)^{\frac{1}{4}} \frac{p_{y i}}{m c k_{p}} \sin \Phi
\end{aligned}
$$

where $x_{i}=r_{i} \cos \theta_{i}, y_{i}=r_{i} \sin \theta_{i}, \frac{p_{x i}}{m c} \approx \sigma a_{i} \cos \left(l \theta_{i}-\hat{\xi}_{i}\right)$, $\frac{p_{y i}}{m c} \approx-a_{i} \sin \left(l \theta_{i}-\hat{\xi}_{i}\right)$, and $a_{i}$ is the laser normalized vector potential when the electrons are ionized, $\Phi \approx \frac{\sqrt{2 \gamma}-\sqrt{2}}{e E_{z} /\left(m c \omega_{p}\right)}$ is the betatron phase, $\gamma=1+\frac{e E_{z}}{m c}\left(z-z_{0}\right)$ is the relativistic factor. Equation (1) can be used to determine $\theta=$ $\operatorname{atan} 2(y, x)[61]$.

The laser pulse transfers part of its angular momentum (spin and orbital) to the ionized electrons as $L_{z} \equiv x_{i} p_{y i}-$ $y_{i} p_{x i} \approx r_{i} a_{i} \sin \left[(l+\sigma) \theta_{i}-\hat{\xi}_{i}\right]$ which is conserved when the electrons move inside an axisymmetric ion column. Since the electrons are ionized uniformly in $\theta_{i}$ by a $C P$ laser, each slice possesses zero net angular momentum if $l+\sigma \neq 0$ with a small spread. On the other hand, when $l+\sigma=0$, each slice possesses a $\xi$-dependent angular momentum. The net transverse momentum of each slice is finite for $l=0$ and zero for $l \neq 0$. Thus, the center of each slice oscillates linearly for $l=0$ and stays at rest for other cases.

To analyze the formation of these twisted structures, we must map the initial spatial distribution of the ionized electrons to their present distribution. For electrons released at $r_{i}, \xi_{i}$ and $t_{i}$, the angular distribution is $g_{l, \sigma}(\theta)=$ $f\left(\theta_{i}\right)\left|\frac{\mathrm{d} \theta}{\mathrm{d} \theta_{i}}\right|^{-1}=\left|\frac{\alpha^{2}+1+2 \sigma \alpha \cos \left[(l+\sigma) \theta_{i}-\hat{\xi}_{i}\right]}{\alpha^{2}-\sigma l-\alpha(l-\sigma) \cos \left[(l+\sigma) \theta_{i}-\hat{\xi}_{i}\right]}\right|$, where $\alpha=\frac{k_{p} r_{i}}{\sqrt{2} a_{i}} \frac{1}{\tan \Phi}$ and $a_{i}$ is assumed to have a weak dependence on $r_{i}$, and $f\left(\theta_{i}\right)=1$ is assumed because a $C P$ laser is used. While $\theta\left(\theta_{i}\right)$ is known, there is no explicit expression for $\theta_{i}$ as a function of $\theta$. However, we can still make some useful observations. Clearly if $l \geq 1$, then if $\alpha=0$ or $\infty, g_{l, \sigma}(\theta)$ is a constant. The variable $\alpha$ evolves as the particles are accelerated and $\alpha=$ $0(\infty)$ when $\Phi=n \pi+\frac{\pi}{2}(n \pi)$ where $n$ is an integer. We next discuss some behaviors for a right-handed $C P$ laser: $g_{l \geq 1,1}(\theta)$ achieves its maxima at $\theta_{i}=\frac{2 n \pi+\hat{\xi}_{i}}{l+1}$ for $\alpha \geq l$ or $0 \leq \alpha<1$, at $\theta_{i}=\frac{(2 n+1) \pi+\hat{\xi}_{i}}{l+1}$ for $\alpha<-l$ or $-1 \leq \alpha<0$, and at a $\theta_{i}$ when the denominator vanishes for $1 \leq|\alpha|<l$. Figure 2(a) shows the dependence of $g_{2,1}$ on $\alpha$ and $\theta_{i}$. The angles $\theta_{i}=\frac{2 n \pi+\hat{\xi}_{i}}{l+1}$ or $\frac{(2 n+1) \pi+\hat{\xi}_{i}}{l+1}$ are mapped to $\theta=\theta_{i}$ when $(\alpha+1) \sin \Phi>0$ and $\theta=\theta_{i}+\pi$ when $(\alpha+1) \sin \Phi<0$. Thus at some betatron phases, these electrons are concentrated at $l+\sigma$ equally spaced angles which depend linearly on $\xi_{i}$. This concentration has a quasiperiod of the betatron phase, $\frac{\pi}{2}$. More details can be found in Appendix G. For these special angles, $g_{l, \sigma}(\theta)$ is then known.

For $l=0$, the dynamics is different, i.e., when $\alpha=0\left(\Phi=n \pi+\frac{\pi}{2}\right)$, then from Eq. (1) it can be seen that
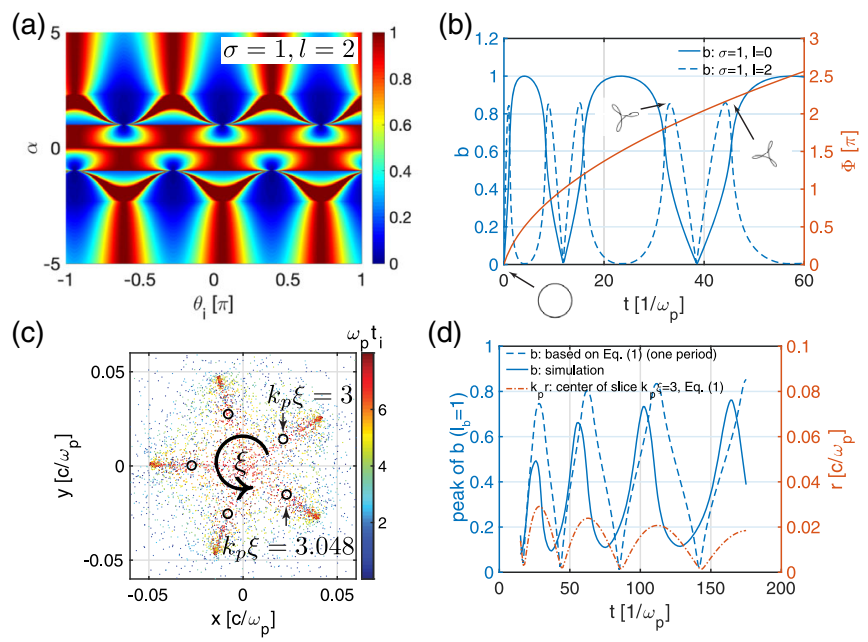

(d)

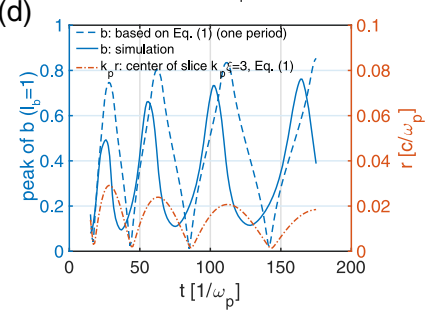

FIG. 2. Theoretical analysis of the twisted structure. (a) $g_{2,1}(\theta)$ with $k_{p} \xi_{i}=2.24$. The value at each $\alpha$ is normalized by 10 when $1 \leq|\alpha|<2$ and its maximum for other $\alpha$. (b) The bunching factor $\left(k=0, l_{b}=l+\sigma\right)$ and the betatron phase of the electrons ionized with $k_{p} r_{i}=0.1, a_{i}=0.1, k_{p} \xi_{i}=2.24$, and $t_{i}=0$. (c) $l=0, \sigma=1$ : the distribution of the electrons and their centers (black circles) at $\omega_{p} t=31$ in five different slices using Eq. (1). (d) The evolution of the peak of the bunching factor and the radial position of the center of the $k_{p} \xi=3$ slice. 
$r=\gamma^{1 / 4} \frac{\sqrt{2} a_{i}}{k_{p}}$ and $\theta=\hat{\xi}_{i}(\sin \Phi>0)$ or $\hat{\xi}_{i}+\pi(\sin \Phi<0)$. This indicates a single spiral beam is formed. Thus, the angular distribution has a quasiperiod of the betatron phase, $\pi$. The bunching factor of the electrons for $l=0$ and $l=2$ in Fig. 2(b) confirms the quasiperiodic behavior of the angle distribution. The insets show the distribution at $\omega_{p} t=0,33$ and 44 for $l=2$.

Physically the electrons in each slice form $l+\sigma$ beamlets and the center of each one conducts linearly oscillations with a $\xi_{i}$-dependent angle. The twisted structures do not rotate, they only flip when the centers cross the origin.

Electrons are ionized at different $r_{i}$, which complicates how electrons are distributed in $\theta$. However, there are always betatron phases where electrons are concentrated at $l+\sigma$ angles due to evolution of $\alpha \propto \frac{1}{\tan \Phi}$. Furthermore, due to longitudinal mixing [30], one slice contains electrons ionized at different times $t_{i}$, which leads to a spread of the phase, which blurs the twisted structure. The betatron phase grows slower as the electrons gain energy, thus the rms spread of the phase decreases as $\sigma_{\Phi} \approx \frac{k_{p} L_{i n j}}{\sqrt{12}} \frac{1}{\sqrt{2 \gamma}}$ [30], where $L_{i n j}$ is the distance over which ionization occurs. In the simulations presented here $k_{p} L_{i n j} \sim 8$. As a result, the amplitude of the oscillations of $b$ increases monotonically during the acceleration as shown in Fig. 2(d). Figure 2(c) shows the distribution of the electrons with different $r_{i}$ and $t_{i}$ at five slices $\left(k_{p} \xi_{i}=2.236,2.249,2,262,2.287,2.3\right.$ which correspond to $k_{p} \xi=3,3.01,3.019,3.029,3.038,3.048$ based on the longitudinal mapping). For each $k_{p} \xi_{i}$, the values of $r_{i}$ and $t_{i}$ are consistent with what is seen in simulations. The concentration at certain angles is clearly seen. In Fig. 2 (d), the long-term behavior of both $b$ and $r$ of the center shows the oscillations and the increase of $b$ with time is seen.

When the laser polarization is left-handed $C P$, similar conclusions can be obtained when $l \neq 1$. However, in the case when $l=1, g_{1,-1}(\theta)$ does not depend on $\theta$, which indicates that the electrons are distributed uniformly in $\theta$, which is consistent with the third row of Fig. 1.

Based on the aforementioned longitudinal mapping [30], the electrons are concentrated at angles that can be written as a function of their positions $\xi$ after injection. For example, when $\alpha<-l$ and $(\alpha+1) \sin \Phi>0\left(\theta_{i}\right.$ where $g_{l, \sigma}$ is maximum is mapped to $\left.\theta=\theta_{i}\right)$,

$$
\theta \approx \frac{2 n+1}{|l+\sigma|} \pi+\frac{\left(k_{L} / k_{p}\right) \sqrt{\left(k_{p} \xi\right)^{2}-4}}{|l+\sigma|},
$$

Eq. (2) indicates there are $|l+\sigma|$ beamlets spiraling around each other. The comparison between the angles at which electrons are concentrated in the $\theta-\xi$ plane and those predicted by Eq. (2) are shown in the second column of Fig. 1. Good agreement is obtained.

The mapping from the phase distribution of LG-CP lasers to the $3 \mathrm{D}$ structure of the injected electrons can be extended to lasers with arbitrary phase distribution and electrons with more complicated structures being produced. Here, we show an example [Fig. 3(a)]: two right-handed $C P$ laser pulses, one with $(l=0, p=0), a_{L}=0.057$, $k_{p} \xi_{\text {center }}=2.5$ and the other with $(l=2, p=0), a_{L}=$ $0.106, k_{p} \xi_{\text {center }}=2.3$, copropagate into the nonlinear wake driven by an $1 \mathrm{GeV}$ electron beam. The structure of the injected electrons gradually evolves from three beamlets at the head of the beam to 1 beamlet at the tail [Fig. 3(b)]. The bunching factor achieves the maximum at $\left(l_{b}=1\right.$, $k \sim 1.7 k_{L}$ ) and their harmonics from the $l=0$ laser, and at $\left(l_{b}=3, k \sim 1.3 k_{L}\right)$ from the $l=2$ laser. Additionally the bunching factor achieves the maximum at $\left(l_{b}=-2, k \approx\right.$ $\left.0.12 k_{L}\right)$ and $\left(l_{b}=4, k \approx 2.8 k_{L}\right)$ which is due to the mutual interactions (beating) between these two laser pulses. The generated electron beam has a charge of $8 \mathrm{pC}$, a $1.2 \mathrm{kA}$ peak current, a 105 (86) $\mathrm{nm}$ emittance and a $1.3 \mathrm{MeV}$ uncorrelated energy spread. By using laser pulses that have different wavelengths, modes, angles, and delays one can produce exotic 3D structured electron beams.

We point out that in contrast to previous work [44,55-57] our work generates electrons with topological structures when they conduct the betatron motion in the linear fields of an ion column. These beams acquire zero net angular momentum with finite spread from the laser. This is different from other work where the beam possesses a significant amount of angular momentum [44,55-57] and the spiral motion of ions is needed to conserve the angular momentum. These high-quality beams are suitable to produce high power coherent radiation with orbital angular momentum (OAM) from ultraviolet to X-ray if they are boosted to high energy and propagate through a magnetic undulator [48].

(a)
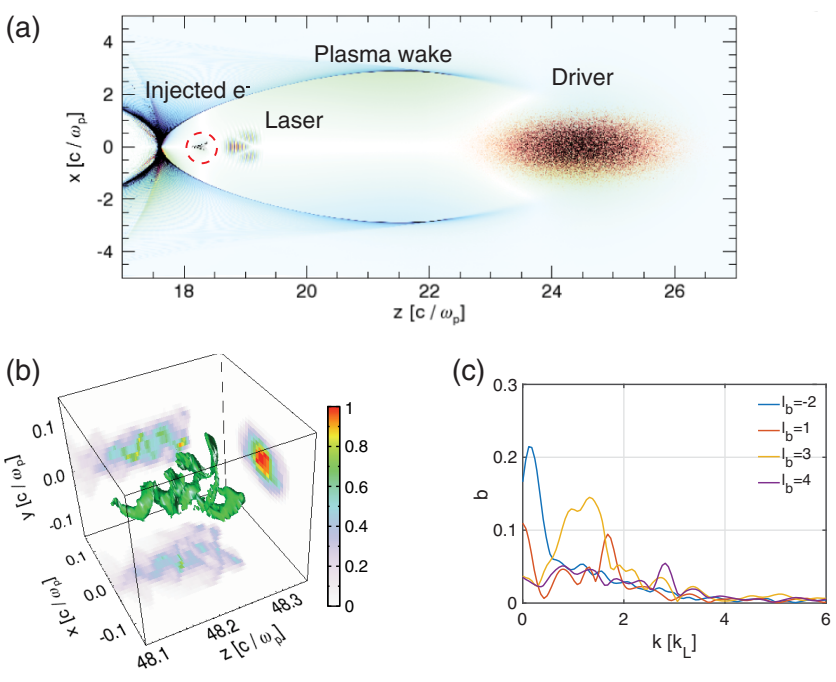

FIG. 3. The structure of the injected electrons when two righthanded $C P$ laser pulses with $(l=0, p=0)$ and $(l=2, p=0)$ are used. (a) A illustration of the laser-triggered ionization injection in a beam driven plasma wake. (b) Density isosurface and its projections to each plane. (c) The bunching factor. The profiles of the pulses are as same as in Fig. 1. 
We point out that in contrast to previous work [44,55-57] we consider using a low-intensity $C P$ laser with LG modes to generate topologically structured electron beams with high quality, i.e., high current, low-energy spread and low emittance. The beams form the topological structures gradually after they leave the laser pulse and conduct the betatron motion in the linear focusing fields inside an ion column. They acquire zero net angular momentum with finite spread (see Appendix F) from the laser which makes it possible to couple them out of the plasma while preserving their structures [66-70]. This is different from other work where the beam possesses a significant amount of angular momentum [44,55-57] and the spiral motion of ions is needed to conserve the angular momentum. These high-quality beams are suitable to produce high-power coherent radiation with OAM from ultraviolet to X-ray wavelengths if they are boosted to high energy in an acceleration section and then propagate through a magnetic undulator [48].

\section{ACKNOWLEDGMENTS}

This work was supported by the U.S. Department of Energy under Contracts No. DE-AC02-76SF00515 and No. DE-SC0010064, the U.S. National Science Foundation under Grants No. 2108970 and No. 1806046, and the DOE Scientific Discovery through Advanced Computing (SciDAC) program through a Fermi National Accelerator Laboratory (FNAL) subcontract No. 644405. The simulations were performed on the resources of the National Energy Research Scientific Computing Center (NERSC), a U.S. Department of Energy Office of Science User Facility located at Lawrence Berkeley National Laboratory, through an ALCC grant. J. V. acknowledges the support of FCT (Portugal) Grant No. SFRH/IF/01635/2015.

\section{APPENDIX A: EXPRESSIONS OF THE LASERS WITH LAGUERRE-GAUSSIAN MODES}

The transverse components of the normalized vector potential of a circularly polarized LG laser used in the simulation is

$\vec{A}(r, \theta, z, t)=-\operatorname{Re}\left(\sigma \vec{e}_{x}+i \hat{e}_{y}\right) a_{L} c_{p}^{|l|}(r, \theta, z) \mathrm{e}^{i(l \theta-k z+\omega t)}$,

where $\hat{e}_{x, y}$ are the unit vector along the $x$ and $y$ directions, $\sigma=1$ for right-handed polarization and $\sigma=-1$ for lefthanded polarization. The LG modes are defined as

$$
\begin{aligned}
c_{p}^{|l|}(r, \theta, z)= & \sqrt{\frac{2 p !}{\pi(p+|l|) !}} \frac{w_{0}}{w(z)}\left(\frac{\sqrt{2} r}{w(z)}\right) \exp \left(-\frac{r^{2}}{w^{2}(z)}\right) \\
& \times L_{p}^{|l|}\left(\frac{2 r^{2}}{w^{2}(z)}\right) \exp \left(-i k_{L} \frac{r^{2}}{2 R(z)}\right) \exp (i \psi(z)),
\end{aligned}
$$

where $L_{p}^{l}$ are the generalized Laguerre polynomials, $w(z)=$ $w_{0} \sqrt{1+\left(\frac{z}{z_{R}}\right)^{2}}$ is the spot size, $R(z)=z\left[1+\left(\frac{z}{z_{R}}\right)^{2}\right]$ is the radius of curvature of the wavefront, $z_{R}=\frac{\pi w_{0}^{2}}{\lambda_{L}}$ is the Rayleigh length, and $\psi(z)=(2 p+|l|+1) \arctan \left(\frac{z}{z_{R}}\right)$. The amplitude is determined through $a_{L}$. These expressions can be found easily in textbooks.

\section{APPENDIX B: PARTICLE-IN-CELL SIMULATION SETUP}

For the simulations shown in Figs. 1, 2, and 4, we use a moving window propagating at speed of light in vacuum $c$ with a box size of $1.3 \times 2.4 \times 2.4\left(c / \omega_{p}\right)^{3}$ and $650 \times 960 \times$ 960 cells along the $z, x$, and $y$ directions, respectively. The cell sizes correspond to $0.002 c / \omega_{p}$ along the $z$ direction and $0.0025 c / \omega_{p}$ along the $x$ and $y$ directions. The time step is $\mathrm{d} t=0.0013245 \omega_{p}^{-1}$ which is close to the Courant limit and one macroparticle per cell is used to represent the $\mathrm{He}^{1+}$ ion. The ions are immobile in the simulations. The code uses the Ammosov-Delone-Krainov (ADK) tunneling ionization model [60].

The self-consistent simulation shown in the second row of Fig. 1 (dashed line in the bunching factor plot) and Fig. 3 uses a moving window propagating at $c$ with a box size of $10 \times 10 \times 10\left(c / \omega_{p}\right)^{3}$ and $5000 \times 1000 \times 1000$ cells along the $z, x$, and $y$ directions, respectively. The grid size is $0.002 c / \omega_{p}$ along the $z$ direction and $0.01 c / \omega_{p}$ along
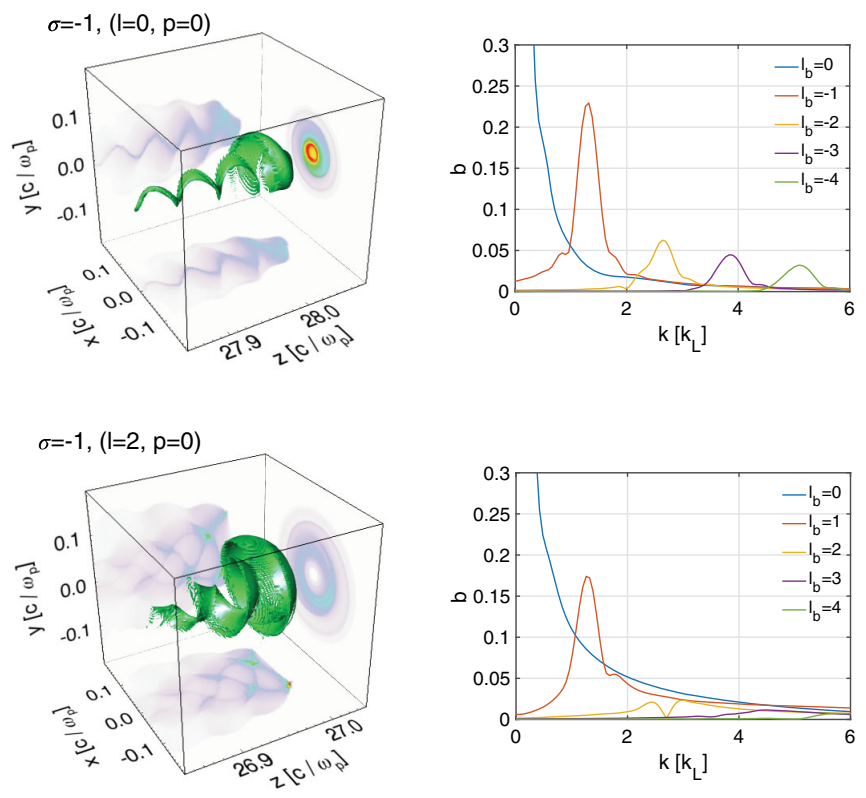

FIG. 4. The structures of the injected electrons at $\omega_{p} t=31$ when a $C P$ laser pulse with $(\sigma=-1, l=0, p=0)$ or $(\sigma=$ $-1, l=2, p=0)$ is used. The first column: the isosurface of the electron density distribution and its projections on each plane. The second column: the bunching factor of the injected electrons. 
the $x$ and $y$ directions. The time step is $\mathrm{d} t=0.001 \omega_{p}^{-1}$ and one macroparticle per cell is used to represent the beam driver electron, the plasma electrons and the $\mathrm{He}^{1+}$ ion. The ions are immobile in the simulations. We use the customized field solver described in Ref. [59]. An electron beam driver with $1 \mathrm{GeV}$ energy and $19 \mathrm{kA}$ peak current is used to excite the nonlinear wave wake The spot size of the bi-Gaussian beam driver $n_{b} \sim \exp \left(-\frac{r^{2}}{2 \sigma_{r}^{2}}-\frac{z^{2}}{2 \sigma_{z}^{2}}\right)$ is $\sigma_{r}=0.7 c / \omega_{p}(8.9 \mu \mathrm{m})$ and its duration is $\sigma_{z}=0.835 c / \omega_{p}(10.6 \mu \mathrm{m})$.

\section{APPENDIX C: THE STRUCTURES OF THE INJECTED ELECTRONS WHEN A LASER PULSE WITH $(\sigma=-1, l=0, p=0)$ OR $(\sigma=-1, l=2, p=0)$ IS USED}

The density isosurface of the injected electrons and their bunching factors in two more cases are shown in Fig. 4: $(\sigma=-1, l=0, p=0)$ and $(\sigma=-1, l=2, p=0)$. Their structures and the maximums of the bunching factor are consistent with the theoretical predictions: when $(\sigma=-1, l=0, p=0)$, a single spiral beam is formed, and the bunching factor achieves its maximum at $\left(l_{b}=\right.$ $\left.-1, k \approx 1.3 k_{L}\right)$ and the beam is rich in spatial harmonics; when $(\sigma=-1, l=2, p=0)$, a single spiral beam is produced and the bunching factor achieves its maximum at $\left(l_{b}=1, k \approx 1.3 k_{L}\right)$. The profile and the intensity of the lasers are the same as that of Fig. 1.

\section{APPENDIX D: LONGITUDINAL PHASE SPACE OF THE INJECTED BEAMS}

In Fig. 5, we show the charge distribution of the injected beams in their longitudinal phase space $\left(\gamma m c^{2}, \xi\right)$ for the five cases studied in Fig. 1. The self-consistent simulation (bottom right) has an acceleration gradient with a smaller $\operatorname{chirp}\left(F_{z} \approx 0.4 \xi m \omega_{p}^{2}\right)$ due to a relatively weak driver. Thus, the beam from this simulation has lower energy at the same acceleration distance.
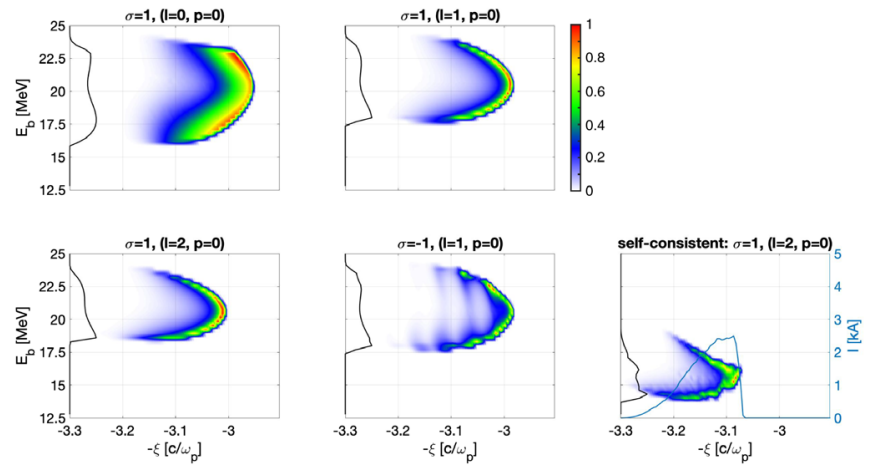

FIG. 5. Longitudinal phase space of the injected beams at $\omega_{p} t=31$. The charge density is normalized to its peak in each subplot. The black lines are the energy spectrum. The blue line in the self-consistent case (bottom right) shows the current profile.
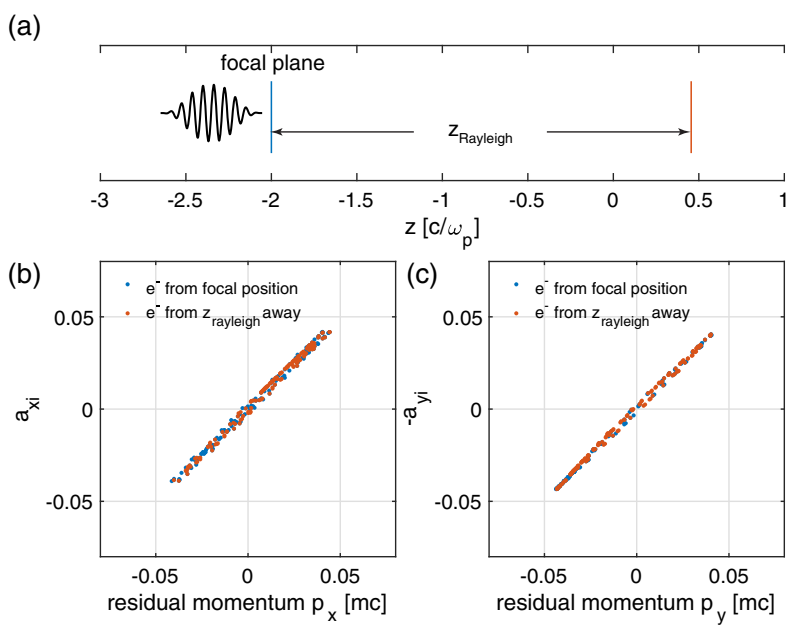

FIG. 6. Simulation study of the conservation of $\vec{P}_{\perp}$. The profile and intensity of the laser pulse is the same as that of Fig. 1.

\section{APPENDIX E: CONSERVATION OF $\overrightarrow{\boldsymbol{P}}_{\perp}$ FOR A FOCUSED LASER WITH LG MODE}

We confirm the conservation of $\vec{P}_{\perp}$ when the electrons are ionized and move inside a focused laser with LG mode by carrying out OSIRIS simulation. The setup of the simulation is shown in Fig. 6(a) where a laser pulse with $(\sigma=1, l=2, p=0)$ is considered. Two lines of $\mathrm{He}^{1+}$ ions at $y=0$ and different axial locations (at the focal position of the laser and one Rayleigh length away from the focal position) are initialized. There is no external field in the simulation, thus the released electrons oscillate inside the laser pulse and drift after the laser passes them. Their momenta after being passed by the laser are the residual momenta we require. As shown in Figs. 6(b) and 6(c), we can see the plane wave (transverse invariance) approximation works well for the parameters studied here.

\section{APPENDIX F: THE TRANSVERSE MOMENTUM DIRECTION OF THE INJECTED BEAMS}

We show the transverse momentum direction of 1000 sampled electrons in Fig. 7 for $(\sigma=1, l=2, p=0)$ case

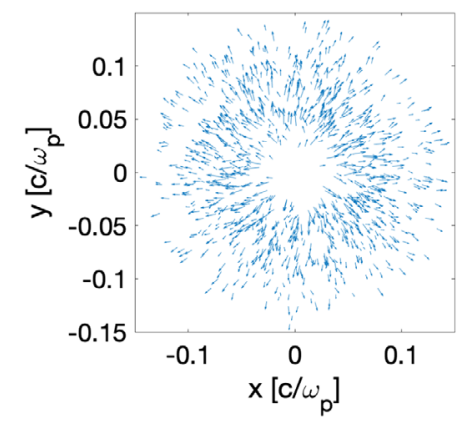

FIG. 7. The direction of the transverse momentum of 1000 sampled electrons. 
at $\omega_{p} t=31$. The direction of the momentum is approximately along the radial direction which indicate each electron has a small angular momentum.

\section{APPENDIX G: PROPERTIES OF $\boldsymbol{g}_{l, \sigma}\left(\boldsymbol{\theta}\left(\boldsymbol{\theta}_{i}\right)\right)$}

In this section, we discuss the properties of the function $g_{l, \sigma}\left(\theta\left(\theta_{i}\right)\right)$. We start with

$$
\tan \theta=\frac{y}{x}
$$

then

$$
\begin{aligned}
\frac{\mathrm{d} \tan \left(\theta\left(\theta_{i}\right)\right)}{\mathrm{d} \theta_{i}} & =\frac{\mathrm{d} \tan \theta}{\mathrm{d} \theta} \frac{\mathrm{d} \theta}{\mathrm{d} \theta_{i}}=\frac{\frac{\mathrm{d} y}{\mathrm{~d} \theta_{i}} x-\frac{\mathrm{d} x}{\mathrm{~d} \theta_{i}} y}{x^{2}} \\
\Rightarrow \frac{\mathrm{d} \theta_{i}}{\mathrm{~d} \theta} & =\frac{\frac{\mathrm{d} \tan \theta}{\mathrm{d} \theta} x^{2}}{\frac{\mathrm{d} y}{\mathrm{~d} \theta_{i}} x-\frac{\mathrm{d} x}{\mathrm{~d} \theta_{i}} y} \\
& =\frac{x^{2}+y^{2}}{\frac{\mathrm{d} y}{\mathrm{~d} \theta_{i}} x-\frac{\mathrm{d} x}{\mathrm{~d} \theta_{i}} y}
\end{aligned}
$$

where $\frac{\mathrm{d} \tan \theta}{\mathrm{d} \theta}=\frac{1}{\cos ^{2} \theta}=\frac{x^{2}+y^{2}}{x^{2}}$ is used. Substituting the expressions of $x$ and $y$, it is straightforward to obtain

$$
\frac{\mathrm{d} \theta_{i}}{\mathrm{~d} \theta}=\frac{\alpha^{2}+1+2 \sigma \alpha \cos \left[(l+\sigma) \theta_{i}-\hat{\xi}_{i}\right]}{\alpha^{2}-\sigma l-\alpha(l-\sigma) \cos \left[(l+\sigma) \theta_{i}-\hat{\xi}_{i}\right]}
$$

where $\alpha=\frac{k_{p} r_{i}}{\sqrt{2} a_{i}} \frac{1}{\tan \Phi}$.

\section{Right-handed case: $\sigma=1$}

When the laser is right-handed $C P$ polarized, the expression for $g_{l, 1}$ can be obtained as

$$
g_{l, 1}(\theta)=\left|\frac{\mathrm{d} \theta_{i}}{\mathrm{~d} \theta}\right|=\frac{\left|\alpha^{2}+1+2 \alpha \cos \bar{x}\right|}{\left|\alpha^{2}-l-\alpha(l-1) \cos \bar{x}\right|},
$$

where $\bar{x}=(l+1) \theta_{i}-\hat{\xi}_{i}$ and $l=0,1,2,3, \ldots$.

When $l=0, g_{l, 1}$ is simplified to $g_{0,1}=\frac{\left|\alpha^{2}+1+2 \alpha \cos \bar{x}\right|}{\left|\alpha^{2}+\alpha \cos x\right|}$. It is easy to see $g_{0,1}$ reaches its maximum at $\bar{x}=2 n \pi$ for $\alpha \geq 1$, at $\bar{x}= \pm \cos ^{-1}(-\alpha)+2 n \pi$ for $-1 \leq \alpha<1$, and at $\bar{x}=$ $(2 n+1) \pi$ for $\alpha<-1$, where $n$ is an integer.

For $l \geq 1$, the story is more complicated. For $\alpha \geq l, g_{l, 1}$ achieves its maximum when $\bar{x}=2 n \pi$ since the denominator is minimum and the numerator is maximum. For $1 \leq \alpha<l$, the denominator is zero when $\bar{x}= \pm \cos ^{-1}\left(\frac{\alpha^{2}-l}{\alpha(l-1)}\right)+2 n \pi$, thus $g_{l, 1}$ reaches its maximum, which is infinity, at these locations. For $0 \leq \alpha<1$, by solving $\mathrm{d} g_{l, 1}(\bar{x}) / \mathrm{d} \bar{x}=0$, the maximum value of $g_{l, 1}$ is found to occur at $\bar{x}=2 n \pi$. The properties of $g_{l, 1}$ for $\alpha<0$ can be found similarly, i.e., $g_{l, 1}$ reaches its maximum at $\bar{x}=(2 n+1) \pi$ for $-1 \leq \alpha<0$ or $\alpha<-l$, and at $\bar{x}= \pm \cos ^{-1}\left(\frac{\alpha^{2}-l}{\alpha(l-1)}\right)+(2 n+1) \pi$ for $-l \leq \alpha<-1$.

\section{Left-handed case: $\sigma=\mathbf{- 1}$}

When the laser is left-handed $C P$ polarized, the expression of $g_{l,-1}$ is

$$
g_{l,-1}(\theta)=\frac{\left|\alpha^{2}+1-2 \alpha \cos \bar{x}\right|}{\left|\alpha^{2}+l-\alpha(l+1) \cos \bar{x}\right|}
$$

where $\bar{x}=(l-1) \theta_{i}-\hat{\xi}_{i}$ and $l=0,1,2,3, \ldots$

When $l=0, g_{l,-1}$ is simplified as $g_{0,-1}=\frac{\left|\alpha^{2}+1-2 \alpha \cos x\right|}{\left|\alpha^{2}-\alpha \cos x\right|}$. It is easy to see $g_{l,-1}$ reaches its maximum at $\bar{x}=(2 n+1) \pi$ for $\alpha \geq 1$, at $\bar{x}= \pm \cos ^{-1} \alpha+2 n \pi$ for $-1 \leq \alpha<1$, and at $x=2 n \pi$ for $\alpha<-1$. When $l=1$, it is trivial to see $g_{1,-1}=1$.

When $l>1$, we need to be more careful. For $\alpha \geq l$, by solving $\mathrm{d} g_{l,-1}(\bar{x}) / \mathrm{d} \bar{x}=0$, the maximum value of $g_{l,-1}$ is found at $\bar{x}=2 n \pi$. When $1 \leq \alpha<l$, the denominator is zero when $\bar{x}= \pm \cos ^{-1}\left(\frac{\alpha^{2}+l}{\alpha(l+1)}\right)+2 n \pi$, thus $g_{l,-1}$ reaches its maximum of infinity at these locations. For $0 \leq \alpha<1, g_{l,-1}$ reaches its maximum at $\bar{x}=(2 n+1) \pi$. The properties of $g_{l,-1}$ for $\alpha<0$ can be found similarly, i.e., $g_{l,-1}$ reaches its maximum at $\bar{x}=(2 n+1) \pi$ for $\alpha<-l$, at $\bar{x}=$ $\pm \cos ^{-1}\left(\frac{\alpha^{2}+l}{\alpha(l+1)}\right)+2 n \pi$ for $-l \leq \alpha<-1$, and at $\bar{x}=2 n \pi$ for $-1 \leq \alpha<0$. The value of $g_{l, \sigma}(\bar{x})$ is shown in Fig. 8 for 5 cases.

\section{Mapping between $\theta_{i}$ and $\theta$ : Right-handed case $\boldsymbol{\sigma}=\mathbf{1}$}

When the laser is right-handed polarized, the relation between $\theta$ and $\theta_{i}$ is

$$
\begin{gathered}
\theta=\operatorname{atan} 2\left(\frac{k_{p} r_{i}}{\sqrt{2} a_{i}} \sin \theta_{i} \cos \Phi-\sin \left(l \theta_{i}-\hat{\xi}_{i}\right) \sin \Phi,\right. \\
\left.\frac{k_{p} r_{i}}{\sqrt{2} a_{i}} \cos \theta_{i} \cos \Phi+\cos \left(l \theta_{i}-\hat{\xi}_{i}\right) \sin \Phi\right) .
\end{gathered}
$$

We can note when $(l+1) \theta_{i}-\hat{\xi}_{i}=2 n \pi, \theta_{i}$ is mapped to

$\theta=\theta_{i}=\frac{2 n \pi+\hat{\xi}_{i}}{l+1} \quad$ if $\frac{k_{p} r_{i}}{\sqrt{2} a_{i}} \cos \Phi+\sin \Phi>0$,

and to

$$
\begin{gathered}
\theta=\pi+\theta_{i}=\frac{(2 n+l+1) \pi+\hat{\xi}_{i}}{l+1} \\
\text { if } \frac{k_{p} r_{i}}{\sqrt{2} a_{i}} \cos \Phi+\sin \Phi<0 .
\end{gathered}
$$

Clearly, the angle $\frac{2 n \pi+\hat{\xi}_{i}}{l+1}$ is equivalent to $\frac{(2 n+l+1) \pi+\hat{\xi}_{i}}{l+1}$ when $l$ is odd. 

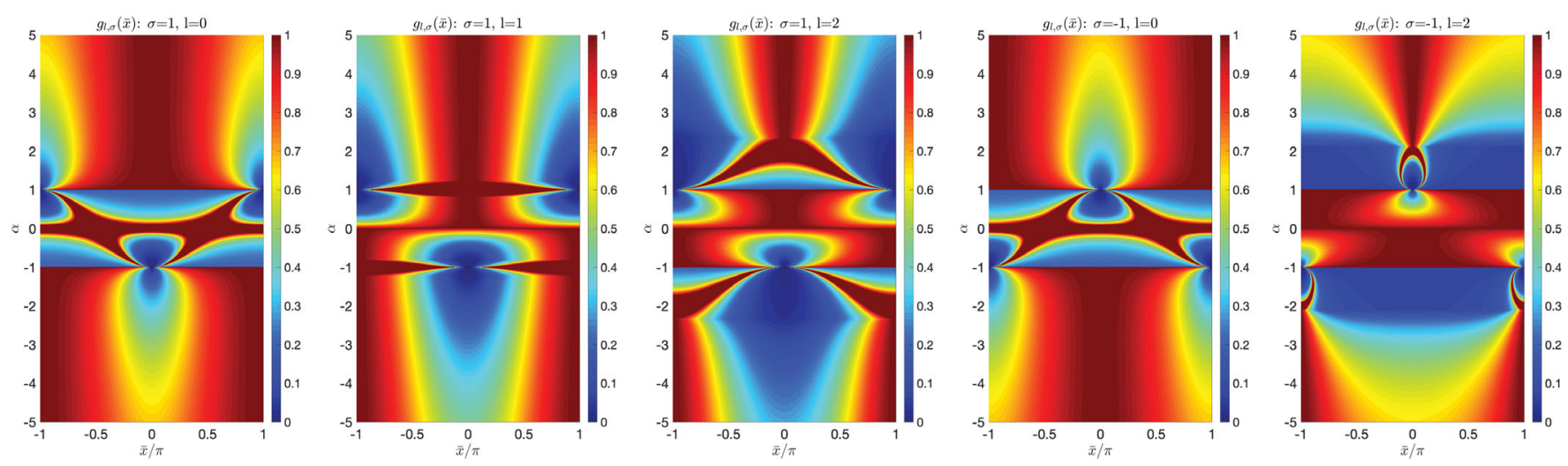

FIG. 8. The value of $g_{l, \sigma}(\bar{x})$. Note the value at each $\alpha$ is normalized by 10 when $1 \leq|\alpha|<l$ and its maximum for other $\alpha$.

When $(l+1) \theta_{i}-\hat{\xi}_{i}=(2 n+1) \pi$,

$\theta=\theta_{i}=\frac{(2 n+1) \pi+\hat{\xi}_{i}}{l+1} \quad$ if $\frac{k_{p} r_{i}}{\sqrt{2} a_{i}} \cos \Phi-\sin \Phi>0$,

and to

$$
\begin{gathered}
\theta=\pi+\theta_{i}=\frac{(2 n+l+2) \pi+\hat{\xi}_{i}}{l+1} \\
\text { if } \frac{k_{p} r_{i}}{\sqrt{2} a_{i}} \cos \Phi-\sin \Phi<0 .
\end{gathered}
$$

These angles are also equivalent when $l$ is odd.

\section{Mapping between $\theta_{i}$ and $\theta$ : Left-handed case $\sigma=-1$}

When the laser is left-handed polarized, the relation between $\theta$ and $\theta_{i}$ is

$$
\begin{gathered}
\theta=\operatorname{atan} 2\left(\frac{k_{p} r_{i}}{\sqrt{2} a_{i}} \sin \theta_{i} \cos \Phi-\sin \left(l \theta_{i}-\hat{\xi}_{i}\right) \sin \Phi,\right. \\
\left.\frac{k_{p} r_{i}}{\sqrt{2} a_{i}} \cos \theta_{i} \cos \Phi-\cos \left(l \theta_{i}-\hat{\xi}_{i}\right) \sin \Phi\right) .
\end{gathered}
$$

We can note when $(l-1) \theta_{i}-\hat{\xi}_{i}=2 n \pi, \theta_{i}$ is mapped to

$\theta=\theta_{i}=\frac{2 n \pi+\hat{\xi}_{i}}{l-1} \quad$ if $\frac{k_{p} r_{i}}{\sqrt{2} a_{i}} \cos \Phi-\sin \Phi>0$,

and to

$$
\begin{gathered}
\theta=\pi+\theta_{i}=\frac{(2 n+l-1) \pi+\hat{\xi}_{i}}{l-1} \\
\text { if } \frac{k_{p} r_{i}}{\sqrt{2} a_{i}} \cos \Phi-\sin \Phi<0 .
\end{gathered}
$$

When $l$ is odd, these angles are equivalent.

When $(l-1) \theta_{i}-\hat{\xi}_{i}=(2 n+1) \pi$,

$$
\theta=\theta_{i}=\frac{(2 n+1) \pi+\hat{\xi}_{i}}{l-1} \text { if } \frac{k_{p} r_{i}}{\sqrt{2} a_{i}} \cos \Phi+\sin \Phi>0,
$$

and to

$\theta=\pi+\theta_{i}=\frac{(2 n+l) \pi+\hat{\xi}_{i}}{l-1}$ if $\frac{k_{p} r_{i}}{\sqrt{2} a_{i}} \cos \Phi+\sin \Phi<0$,

when $l$ is odd, these angles are also equivalent.

\section{An example of the evolution of the angles}

We show the evolution of $\theta$ for particles with different $\theta_{i}$ when $(l=2, \sigma=1)$ in Fig. 9. When there are three knots, i.e., the angles concentrate at three distinct values, the bunching factor reaches its maximum [see Fig. 2(b) in the main text].
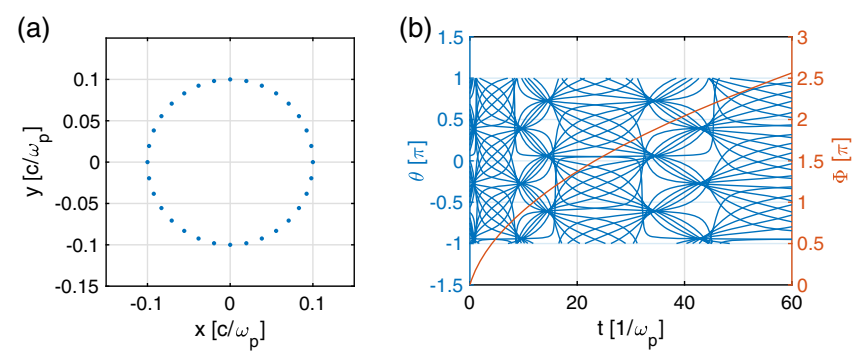

FIG. 9. Left: the initial distribution of the electrons. These electrons are initialized with $k_{p} r_{i}=0.1, a_{i}=0.1, k_{p} \xi_{i}=2.24$ and $t_{i}=0$. Right: the evolution of $\theta$ and the betatron phase $\Phi$. 
[1] T. Tajima and J. M. Dawson, Phys. Rev. Lett. 43, 267 (1979).

[2] P. Chen, J. Dawson, R. W. Huff, and T. Katsouleas, Phys. Rev. Lett. 54, 693 (1985).

[3] C. Joshi, S. Corde, and W. Mori, Phys. Plasmas 27, 070602 (2020).

[4] I. Blumenfeld et al., Nature (London) 445, 741 (2007).

[5] A. J. Gonsalves et al., Phys. Rev. Lett. 122, 084801 (2019).

[6] E. Adli et al., Nature (London) 561, 363 (2018).

[7] W. Wang et al., Nature (London) 595, 516 (2021).

[8] J. M. Cole et al., Phys. Rev. X 8, 011020 (2018).

[9] K. Poder et al., Phys. Rev. X 8, 031004 (2018).

[10] S. Corde, K. T. Phuoc, G. Lambert, R. Fitour, V. Malka, A. Rousse, A. Beck, and E. Lefebvre, Rev. Mod. Phys. 85, 1 (2013).

[11] K. Behm et al., High Energy Density Phys. 35, 100729 (2020).

[12] J. Cole et al., Sci. Rep. 5, 13244 (2015).

[13] B. Hidding, G. Pretzler, J. B. Rosenzweig, T. Königstein, D. Schiller, and D. L. Bruhwiler, Phys. Rev. Lett. 108, 035001 (2012).

[14] F. Li et al., Phys. Rev. Lett. 111, 015003 (2013).

[15] W. T. Wang et al., Phys. Rev. Lett. 117, 124801 (2016).

[16] X. L. Xu, F. Li, W. An, T. N. Dalichaouch, P. Yu, W. Lu, C. Joshi, and W. B. Mori, Phys. Rev. Accel. Beams 20, 111303 (2017).

[17] T. Dalichaouch, X. Xu, F. Li, A. Tableman, F. Tsung, W. An, and W. Mori, Phys. Rev. Accel. Beams 23, 021304 (2020).

[18] S. Bulanov, N. Naumova, F. Pegoraro, and J. Sakai, Phys. Rev. E 58, R5257 (1998).

[19] H. Suk, N. Barov, J. B. Rosenzweig, and E. Esarey, Phys. Rev. Lett. 86, 1011 (2001).

[20] C. G. R. Geddes, K. Nakamura, G. R. Plateau, C. Toth, E. Cormier-Michel, E. Esarey, C. B. Schroeder, J. R. Cary, and W. P. Leemans, Phys. Rev. Lett. 100, 215004 (2008).

[21] A. Gonsalves et al., Nat. Phys. 7, 862 (2011).

[22] A. Buck et al., Phys. Rev. Lett. 110, 185006 (2013).

[23] A. Deng et al., Nat. Phys. 15, 1156 (2019).

[24] M. Chen, Z.-M. Sheng, Y.-Y. Ma, and J. Zhang, J. Appl. Phys. 99, 056109 (2006).

[25] E. Oz et al., Phys. Rev. Lett. 98, 084801 (2007).

[26] A. Pak, K. A. Marsh, S. F. Martins, W. Lu, W. B. Mori, and C. Joshi, Phys. Rev. Lett. 104, 025003 (2010).

[27] N. Vafaei-Najafabadi et al., Phys. Rev. Lett. 112, 025001 (2014).

[28] O. Lundh et al., Nat. Phys. 7, 219 (2011).

[29] C. Emma, X. Xu, A. Fisher, R. Robles, J. MacArthur, J. Cryan, M. Hogan, P. Musumeci, G. White, and A. Marinelli, APL Photonics 6, 076107 (2021).

[30] X. L. Xu et al., Phys. Rev. Lett. 112, 035003 (2014).

[31] L.-L. Yu, E. Esarey, C. B. Schroeder, J.-L. Vay, C. Benedetti, C. G. R. Geddes, M. Chen, and W. P. Leemans, Phys. Rev. Lett. 112, 125001 (2014).

[32] X. L. Xu et al., Phys. Rev. ST Accel. Beams 17, 061301 (2014).

[33] O. Lundh, C. Rechatin, J. Lim, V. Malka, and J. Faure, Phys. Rev. Lett. 110, 065005 (2013).

[34] X. Xu et al., Phys. Rev. Lett. 117, 034801 (2016).
[35] J. Wenz et al., Nat. Photonics 13, 263 (2019).

[36] A. Lumpkin et al., Phys. Rev. Lett. 125, 014801 (2020).

[37] X. Xu, F. Li, F. S. Tsung, K. Miller, V. Yakimenko, M. J. Hogan, C. Joshi, and W. B. Mori, arXiv:2010.16081.

[38] A. Gover, R. Ianconescu, A. Friedman, C. Emma, N. Sudar, P. Musumeci, and C. Pellegrini, Rev. Mod. Phys. 91, 035003 (2019).

[39] B. Pollock et al., Phys. Rev. Lett. 115, 055004 (2015).

[40] J. Vieira and J. Mendonça, Phys. Rev. Lett. 112, 215001 (2014).

[41] J. T. Mendonça, B. Thidé, and H. Then, Phys. Rev. Lett. 102, 185005 (2009).

[42] J. Vieira, R. M. Trines, E. P. Alves, R. Fonseca, J. Mendonça, R. Bingham, P. Norreys, and L. Silva, Nat. Commun. 7, 10371 (2016).

[43] J. Vieira, R. M. Trines, E. P. Alves, R. Fonseca, J. Mendonça, R. Bingham, P. Norreys, and L. Silva, Phys. Rev. Lett. 117, 265001 (2016).

[44] J. Vieira, J. Mendonça, and F. Quéré, Phys. Rev. Lett. 121, 054801 (2018).

[45] J. Vieira, M. Pardal, J. Mendonça, and R. Fonseca, Nat. Phys. 17, 99 (2021).

[46] L. Allen, M. W. Beijersbergen, R. J. C. Spreeuw, and J. P. Woerdman, Phys. Rev. A 45, 8185 (1992).

[47] A. M. Yao and M. J. Padgett, Adv. Opt. Photonics 3, 161 (2011).

[48] E. Hemsing, A. Knyazik, M. Dunning, D. Xiang, A. Marinelli, C. Hast, and J. B. Rosenzweig, Nat. Phys. 9, 549 (2013).

[49] M. van Veenendaal and I. McNulty, Phys. Rev. Lett. 98, 157401 (2007).

[50] A. Picón, J. Mompart, J. V. de Aldana, L. Plaja, G. Calvo, and L. Roso, Opt. Express 18, 3660 (2010).

[51] A. S. Rury, Phys. Rev. A 87, 043408 (2013).

[52] M. van Veenendaal, Phys. Rev. B 92, 245116 (2015).

[53] N. Jhajj, I. Larkin, E. Rosenthal, S. Zahedpour, J. Wahlstrand, and H. Milchberg, Phys. Rev. X 6, 031037 (2016).

[54] C. Hernández-García, J. Vieira, J. T. Mendonca, L. Rego, J. S. Román, L. Plaja, P. R. Ribic, D. Gauthier, and A. Picón, Photonics, 4, 28 (2017).

[55] C. Liu et al., Phys. Plasmas 23, 093120 (2016).

[56] L. Ju, C. Zhou, K. Jiang, T. Huang, H. Zhang, T. Cai, J. Cao, B. Qiao, and S. Ruan, New J. Phys. 20, 063004 (2018).

[57] C. Baumann and A. Pukhov, Phys. Plasmas 25, 083114 (2018).

[58] R. Fonseca et al., Lect. Notes Comput. Sci. 2331, 342 (2002).

[59] X. Xu, F. Li, F. S. Tsung, T. N. Dalichaouch, W. An, H. Wen, V. K. Decyk, R. A. Fonseca, M. J. Hogan, and W. B. Mori, J. Comput. Phys. 413, 109451 (2020).

[60] M. V. Ammosov, N. B. Delone, and V. P. Krainov, Sov. Phys. JETP 64, 1191 (1986).

[61] The definition of function $\operatorname{atan} 2(y, x)$ can be found in many place, e.g., https://en.wikipedia.org/wiki/Atan2.

[62] N. Jain, T. M. Antonsen, and J. P. Palastro, Phys. Rev. Lett. 115, 195001 (2015). 
[63] G. Stancari, A. Valishev, G. Annala, G. Kuznetsov, V. Shiltsev, D. A. Still, and L. G. Vorobiev, Phys. Rev. Lett. 107, 084802 (2011).

[64] S. Wang et al., Phys. Rev. Lett. 88, 135004 (2002).

[65] X. Xu et al., Phys. Rev. ST Accel. Beams 17, 061301 (2014).

[66] K. Floettmann, Phys. Rev. ST Accel. Beams 17, 054402 (2014).
[67] I. Dornmair, K. Floettmann, and A. R. Maier, Phys. Rev. ST Accel. Beams 18, 041302 (2015).

[68] X. L. Xu et al., Phys. Rev. Lett. 116, 124801 (2016).

[69] R. Ariniello, C. E. Doss, K. Hunt-Stone, J. R. Cary, and M. D. Litos, Phys. Rev. Accel. Beams 22, 041304 (2019).

[70] Y. Zhao, W. An, X. Xu, F. Li, L. Hildebrand, M. J. Hogan, V. Yakimenko, C. Joshi, and W. B. Mori, Phys. Rev. Accel. Beams 23, 011302 (2020). 